\title{
ENTREVISTA A ALBERTO ACOSTA La moratoria petrolífera en la Amazonía ecuatoriana, una propuesta inspiradora para la Cumbre de Copenhague
}

Matthieu Le Quang ${ }^{1}$

\section{El proyecto ITT en Ecuador: dejar el crudo en tierra o el camino hacia otro modelo de desarrollo}

El proyecto ITT (siglas tomadas del nombre de los tres pozos de exploración perforados en la zona: Ishpingo-Tambococha-Tiputini) es una de las iniciativas del gobierno ecuatoriano para luchar contra el calentamiento climático. Se trata de no explotar unos 850 millones de barriles de petróleo situados en el Parque Yasuní, que constituye una reserva natural con una de las biodiversidades más importantes en el mundo. La explotación de este petróleo pesado, de 14 grados API, podría significar para el Estado un ingreso que fluctuaría entre 5.000 y 6.000 millones de dólares (con un precio cercano a 70 dólares el barril).

El Ecuador tiene una economía basada, principalmente, en la renta petrolera. Recuérdese que el petróleo representó el 22,2\% del PIB, el 63,1\% de las exportaciones y el 46,6\% del Presupuesto General del Estado en el año 2008.

Las reservas del ITT representan cerca del 20\% de las reservas totales conocidas en el país, lo que supone una fuente financiera importante en un país tan pobre como el Ecuador. Sin embargo, la propuesta del gobierno ecuatoriano es no explotar esas reservas por diversas razones, no sólo ambientales.

Ecuador, partiendo del principio de corresponsabilidad por los pro-

* Candidato al doctorado en Ciencias Políticas en el Instituto de Estudios Políticos de Aix-en-Provence. Investigador asociado a FLACSO sede Ecuador. Entrevista realizada el jueves 10 de septiembre de 2009, Boletín ECOS n 8, CIP-Ecosocial, Madrid. Alberto Acosta, ex ministro de Energía y Minas de Ecuador y autor (sic) de la propuesta, explica el proyecto ITT

1 Alberto Acosta, economista y profesor-investigador de FLACSO sede Ecuador, es uno de los impulsores del proyecto ITT. Fue presidente de la Asamblea Constituyente, desde noviembre de 2007 hasta junio de 2008; y ministro de Energía y Minas, de enero hasta junio de 2007, posición desde la que por primera vez propuso públicamente esta iniciativa. También es uno de los fundadores de Movimiento País, el movimiento político del Presidente de la República Rafael Correa. En esta entrevista explica los principios que inspiran la propuesta y los beneficios que generaría si la comunidad internacional apoya financieramente la iniciativa. 
blemas ambientales globales pide a cambio a la comunidad internacional una contribución cercana al 50\% de los ingresos que se podrían conseguir si explotara ese petróleo. Es una propuesta que tiene como meta luchar contra del calentamiento climático y contra la pérdida -sin posibilidad de retorno- de una muy rica biodiversidad, impedir la emisión de unas 410 millones de toneladas de $\mathrm{CO}_{2}$; frenar la deforestación y la contaminación de los suelos; así como el deterioro de las condiciones de vida de los habitantes de la región. Además, esta es una manera efectiva de prevenir la transformación de la selva amazónica en una sabana, lo que provocaría una disminución sustancial de la cantidad de agua en todo el continente.

En diciembre próximo, durante la cumbre mundial de Copenhague en la que se reflexionará sobre los alcances y limitaciones del Acuerdo de Kioto, destinado a mitigar e incluso frenar los perniciosos efectos de los cambios climáticos, el Ecuador espera que este proyecto sirva como modelo. El proyecto ITT representa una revolución ecológica que conduce a un modelo alternativo de desarrollo a partir de una economía pospetrolera para el Ecuador y el mundo. Sin embargo, a pesar de los esfuerzos del gobierno ecuatoriano y del presidente Rafael Correa, así como de algunas personas en Ecuador y en otras regiones del planeta, la comunidad internacional tarda en reaccionar y en apoyar este proyecto. Por el momento, sólo Alemania, a través de su Parlamento y su gobierno, se ha comprometido a financiar este proyecto con unos 50 millones de euros al año, por trece años, que es el tiempo que duraría el beneficio que produciría la explotación y exportación del crudo del ITT. Hay otros países, como Noruega, en donde esta iniciativa ha encontrado un eco inicial positivo, así como algunas organizaciones, como la Comunidad de Madrid, además de varias decenas de personalidades en el mundo entero.

Alberto Acosta es uno de los iniciadores de este proyecto. Es economista y profesor-investigador de FLACSO, sede Ecuador. Fue Presidente de la Asamblea Constituyente, desde noviembre de 2007 hasta junio de 2008; y Ministro de Energía y Minas, de enero hasta junio de 2007, posición desde la que por primera vez propuso públicamente esta iniciativa. También es uno de los fundadores de Movimiento País, el movimiento político del presidente de la República Rafael Correa. 
M. L. Q. ¿Cómo surgió la idea del proyecto ITT y cómo se desarrollaron los primeros pasos?

A. A. El proyecto ITT surge como resultado de un esfuerzo colectivo que tiene una larga historia y muchos procesos que fueron convergiendo en un punto dado. Cuando yo presenté por primera vez esta idea, a inicios del 2007, había transcurrido mucho tiempo desde que se comenzó a trabajar en una propuesta que buscaba la moratoria de la actividad petrolera en la Amazonía ecuatoriana. Esto emergió en primer lugar de las luchas de resistencia de los pueblos indígenas, particularmente en el centro sur de la Amazonía, orientadas a impedir que la actividad petrolera se expanda hacia sus territorios, así como de los grupos de colonos mestizos en la Amazonía norte y de los pueblos indígenas afectados por la actividad de la compañía Chevron, antes Texaco. Esto es un primer punto fundamental y que se debe tener en cuenta. Había un proceso de lucha en contra de ese atropello, que se plasma en el juicio contra la Texaco en Ecuador y que constituye un hito en la resistencia de la sociedad amazónica y nacional contra las prácticas depredadoras de las empresas petroleras.
Desde estos procesos sociales en los que intervinieron otros grupos, en particular, Acción Ecológica, se comienza a discutir la posibilidad de una moratoria petrolera. Ya hace muchos años, casi diez, se plasmó esta idea en un libro llamado Ecuador pospetrolero. Entonces se habló sobre la posibilidad de una moratoria petrolera. Gradualmente fuimos concentrando nuestra atención no sólo en impedir la ampliación de la frontera petrolera sino en reforzar las propuestas de conservación y de respeto a los territorios indígenas. Es muy conocido que el Ecuador es privilegiado en términos de biodiversidad. En el sector en donde se pretendía impulsar la actividad petrolera ligada al ITT hay dos áreas de protección especialmente importantes: el Cuyabeno y el Yasuní. Son zonas que tienen una enorme biodiversidad como producto de no haber sido afectadas por la glaciación. Son reservas del Pleistoceno que las hacen especialmente ricas en términos de vida. Cuando se sucede la glaciación, las zonas Norte y Sur del planeta, se ven copadas por el hielo hasta más de la mitad de Europa y todos los Estados Unidos. Entonces, en esta zona central del planeta, se concentró la vida sin sufrir afectación. Es más, desde allí se repobló lo que actualmente 
conocemos como la Amazonía. Por eso se explica esta enorme biodiversidad.

En este punto me interesa recordar que formulamos una estrategia específica para esta región apenas fui nombrado Ministro de Energía y Minas en enero del 2007. Antes de asumir el cargo trabajé esta iniciativa, particularmente con Esperanza Martínez. Ella me presentó un ayuda-memoria donde se discutía esta posibilidad. Luego, ya en el ministerio, pulimos esta propuesta. Así surge esta idea en una primera fase.

Es importante anotar que inicialmente esta propuesta revolucionaria, sin duda alguna, provocó un enfrentamiento dentro del gobierno del presidente Rafael Correa, quien tuvo algunos reparos derivados de las urgencias económicas que tiene un país empobrecido como Ecuador. Por un lado, la propuesta la lideraba yo como Ministro de Energía y Minas. Era una decisión poco entendida y no comprendida por la lógica tradicional. Resultaba inconcebible que el propio ministro del ramo proponga dejar el petróleo en el suelo, no explotarlo. Por otro lado, el presidente de la empresa estatal Petroecuador, quien quería sacar el petróleo del lugar, presionaba dentro y fuera del gobierno para acelerar su explotación. Recuérdese que yo era el Presidente del Directorio de Petroecuador y el colega era Presidente Ejecutivo de dicha empresa. Teníamos una posición enfrentada. Mientras yo buscaba consolidar la no extracción de crudo, el Presidente de la empresa estatal aceleraba los trámites para entregar este yacimiento hidrocarburífero a varias empresas petroleras. Tan es así, que él, sin informarme, negociaba con las empresas estatales de Chile (Enap), China (Sinopec) y Brasil (Petrobras). Conversaba también con los represeantes de la empresa estatal venezolana (PDVSA) para extraer el petróleo. Su meta era llegar a firmar un convenio para extraer rápidamente el petróleo.

La situación era tensa. De tal manera que fuimos a un directorio de Petroecuador, en el que nos reuníamos normalmente muy temprano por la mañana (a las seis de la mañana ya empezaban las sesiones), en la que participó el propio presidente Rafael Correa. El escuchó los argumentos de una y otra parte. Luego optó por apoyar la tesis de dejar el petróleo en tierra, siempre que se tenga una compensación financiera internacional, porque en esa época hablábamos de compensación... Si no había la compensación financiera, se explotaría el petróleo, dijo el 
Presidente. El tema financiero, hay que reconocerlo, estuvo en el centro del debate desde entonces, y sirvió para bajar las tensiones alrededor de que el país perdería mucho si no se extraía el crudo.

Así es que comienza a cristalizarse esta idea en el ámbito gubernamental, que hasta su aprobación hacia medidos del 2009 atravesó por un complejo y muy contradictorio proceso de acercamientos y distanciamientos. En varias oportunidades, sobre todo en el exterior, el presidente Correa apoyó decididamente esta iniciativa, mientras que, en otras ocasiones, puso reparos y dio marcha atrás, frenando el avance del proyecto.

\section{L. Q. El Ecuador necesita el dine-} ro de este petróleo. Las exportaciones del petróleo representan casi la mitad de los recursos del Estado. ¿Cuáles fueron los argumentos para convencer al presidente Correa y a los otros?

A. A. Dentro de este proceso de lucha y de aprendizaje, en el que se combinan la resistencia y la construcción de alternativas, nosotros comprendimos claramente, incluso durante las discusiones llevadas con Rafael Correa antes de ser candidato presidencial, que la extracción de petróleo por sí sola no era suficiente para desarrollar el país.
El Ecuador ya tiene una larga tradición de explotación petrolera en la Amazonía y no se ha desarrollado. Comenzamos a exportar el petróleo amazónico desde 1972; aunque ya mucho antes, a partir de la segunda década del siglo XX, en menor cantidad, habíamos exportado petróleo desde la península de Santa Elena. Desde que fluyó por primera vez el crudo el 23 de marzo de 1967, y más concretamente desde agosto de 1972, cuando zarpó el primer cargamento de petróleo al mercado internacional, se han extraído más de 4.000 millones de barriles de petróleo. El país ha recibido en términos nominales cerca de 90.000 millones de dólares. Y no nos hemos desarrollado.

Entonces, ahí surge la pregunta: ¿conviene seguir haciendo lo mismo? La respuesta es no. La extracción de petróleo en la Amazonía, directa y también indirectamente, condujo a deteriorar las condiciones ambientales y sociales de la población amazónica. Tenemos una tremenda deforestación, erosión, contaminación del suelo, del agua y del aire. El nororiente de la Amazonía ecuatoriana es totalmente diferente de lo que era antes. Yo tuve la oportunidad de estar en esta región en el año 1969. Entonces había selva, ahora ya no hay selva, hay mucho deterioro 
ambiental y también humano. Las provincias amazónicas registran la mayor pobreza de todo el Ecuador, y son las provincias petroleras las más pobres de dicha región.

Asimismo, una continuada deforestación amazónica provoca pérdidas de agua en la sierra ecuatoriana y en el resto del país. Las nubes amazónicas son cada vez menos compactas y esto conduce a una reducción del caudal de agua lluvia.

Adicionalmente, le hicimos comprender al Presidente la importancia que tiene la enorme biodiversidad existente en esa región de la Amazonía. En un solo árbol del Yasuní, puede haber un mayor número de especies de escarabajos nativos que en toda Europa. En una hectárea del Yasuní, hay un mayor número de especies de árboles nativos que en toda América del Norte. Durante la glaciación del planeta, tal como señalé antes, ahí se concentró la vida.

Otro de los argumentos fue que le presentamos razones éticas indiscutibles. Ahí viven pueblos no contactados, pueblos libres en aislamiento voluntario: los Tagaeri, los Taromenane y los Oñamenane -que forman parte de la nacionalidad Waorani-. Por lo tanto, tenemos un reto humano enorme. Cabe mencionar que desde el Ministerio de Energía y Minas, trabajé con el Ministerio de
Ambiente para elaborar una propuesta de política pública para pueblos en aislamiento voluntario.

Anotemos un hecho que no puede quedar al margen: la actividad petrolera de la compañía ChevronTexaco, entre los años sesenta y los noventa del siglo XX, provocó la desaparición de dos pueblos enteros, los Tetetes y los Sansahauris. Las voces, las risas, los comentarios, los juegos, los cantos, los lloros de esos pobladores nunca más los vamos a volver a oír. Desaparecieron todos. Fue un choque con la "cultura occidental y cristiana", que liquidó a estos pueblos. Para completar esta tragedia, de manera irónica, los nombres de estos dos pueblos desaparecidos sirven para denominar dos campos petroleros en el norte de la Amazonía ecuatoriana, donde habitaban estos pueblos...

Es más, sobre Texaco pesan también todos los daños económicos, sociales y culturales causados a los indígenas siona, secoya, cofán, kichwa y waorani, además de perjuicio a los colonos blanco-mestizos. En el ámbito psicosocial las denuncias son múltiples: violencia sexual por parte de los operadores de la compañía contra mujeres adultas y menores de edad mestizas e indígenas; abortos espontáneos; discriminación y racismo; desplazamientos forzados, noci- 
vo impacto cultural y ruptura de la cohesión social.

Y por último, extraer ese petróleo no sólo que afectaría a la biodiversidad y la vida -que ya es motivo suficiente para no hacerlo - sino que provocaría una emisión de unas 410 millones toneladas de $\mathrm{CO}_{2}$. Es un petróleo pesado, que tiene mucho azufre, que tiene que ser descontaminado. Eso costaría a la humanidad mucho dinero, porque eso no le cuesta sólo al Ecuador. Va a costar, sobre todo, a los países ricos, que son los que más han contaminado y que están preocupados por mitigar dicha contaminación.

Todos esos elementos, sumados a la necesidad de pensar en una economía pospetrolera, considerando que las reservas petroleras en el país se acaban, fueron configurando un escenario para exigir la no-explotación del ITT.

Todo lo anterior se complementó con reflexiones tendientes a plantear una nueva modalidad de acumulación, orientada a construir un esquema pospetrolero, es decir una economía posextractivista. Ecuador, como casi todos los países que han pretendido desarrollarse a partir de la exportación del petróleo y también de los recursos minerales, ha transitado por una senda muy compleja. Han consolidado economías rentis- tas, sociedades clientelares y gobiernos de tinte autoritario. Es una cuestión que todavía está en disputa dentro del gobierno del presidente Correa y, también, en los gobiernos considerados como progresistas en la región.

M. L. Q. Este proyecto se sirve de los mecanismos creados por el Protocolo de Kioto. Pero en su visión va más allá de los objetivos de este acuerdo internacional porque la meta no sólo es hacer pagar a los que contaminan sino también proteger la biodiversidad, en una parte importante de la Amazonía. ¿Puedes explicar los objetivos y los mecanismos de este proyecto?

A. A. El proyecto supera la visión y mecanismos del Protocolo de Kioto, si bien surge como una propuesta de compensación. Al menos la mitad de los ingresos que corresponderían a una factura de una posible explotación, se situarían por fuera de los mecanismos de Kioto, porque entre otras cosas, éstos están orientados a la absorción de emisiones. En este caso no hablamos de absorber emisiones, sino de evitarlas. Aun cuando hubo intenciones de vincular totalmente el proyecto al mercado existente del carbono, esta pretensión no prosperó. 
Creemos, además, que el proteger la vida no se lo puede hacer recurriendo a relaciones mercantiles. Ponerle un valor monetario a la naturaleza, en otras palabras a la vida, no nos parece que es lo más adecuado. Entonces, la propuesta ITT ya de por sí rebasa la esencia del mercado de carbono, la esencia del Protocolo de Kioto. Por ello este proyecto se enmarca en una lógica posKioto. Nosotros creemos que eso tiene que ser uno de los elementos consustanciales, fundamentales para avanzar en la construcción de respuestas realmente efectivas a las demandas que se derivan de los cambios climáticos que afectan a la humanidad.

Con esta propuesta de no explotación de crudo pretendíamos, además, replantear una agenda internacional sobre cambios climáticos: hablar del petróleo y su excesivo consumo como principal agente de estos fenómenos, impulsar acciones prácticas en donde se pongan en juego las responsabilidades comunes pero diferenciadas, posicionar en la agenda internacional global la importancia de la conservación de biodiversidad y del respeto a los derechos de los pueblos indígenas.

Nosotros -me refiero sobre todo al trabajo que hemos realizado en este año con Eduardo Gudynas (uruguayo), Joseph Vogel (norteamerica- no), Esperanza Martínez y yo (ecuatorianos)- hemos desarrollado una línea diferente. Hablamos de co-responsabilidad y de política de Estado. No creemos que se pueda solicitar una compensación por la compensación. Aquí hay una co-responsabilidad compartida, en especial de parte de las sociedades más ricas que son las que más han destrozado el planeta. El gobierno ecuatoriano, asimismo, tiene que transformar esta decisión en una política estatal, independiente haya o no el financiamiento que se busca.

Insisto, la compensación, en realidad, no es sino una contribución a partir del principio de co-responsabilidad de los países que han deteriorado el medio ambiente -Estados Unidos, Europa y Japón-. Esa es la idea medular.

El gobierno nacional en su propuesta ha recogido muchos de estos criterios presentados por el grupo mencionado, y todavía mantiene la opción del mercado de carbono para los recursos adicionales que se obtengan. Se colocarían Certificados de Garantía Yasuní y eso generaría réditos. Además, Ecuador, con esos réditos, financiaría proyectos de reforestación, proyectos para el desarrollo de energías alternativas renovables y otros proyectos más. Y a cuenta de estos proyectos, aspiraría a conseguir 
recursos en el mercado de carbono. Es decir, se busca obtener los recursos para mantener el crudo en tierra y no emitir el $\mathrm{CO}_{2}$, al tiempo que los réditos que estos recursos generen servirían para hacer inversiones que le permitirían operar en el mercado de carbono, una cuestión con la que no estamos totalmente convencidos.

\section{L. Q. ¿De qué servirá el fondo eco-} nómico que el Ecuador tendría y sobre todo quién decidirá cómo se podría utilizar?

A. A. La definición de quién usa el fondo está muy clara: tiene que ser el Estado ecuatoriano. Es indudable. Y en cuanto al manejo del fondo, en qué se usan los recursos, lo decide el Estado ecuatoriano. ¿Cómo se maneja el fondo? Eso es un tema de discusión, que debe ser llevada a cabo por el gobierno nacional y la sociedad ecuatoriana, particularmente, con las poblaciones afectadas por las actividades petroleras, y los pueblos del Yasuní, con el fin de avanzar colectivamente en la construcción de un Ecuador pospetrolero, que fue la idea fundacional del proyecto.

La administración de la cuenta, es decir del fideicomiso, debe estar a cargo de alguna instancia de Naciones Unidas. No puede ser un banco multilateral, como el BID (Banco
Interamericano de Desarrollo), tal como se pretendía desde el equipo gubernamental encargado de llevar adelante este proyecto. Queremos que sea Naciones Unidas porque este proyecto puede ser reproducido, replicado en otras partes.

El destino de los recursos -tema en discusión- sería para reforestación; para fuentes alternativas de energía; para proyectos sociales en educación, salud; para la restauración ambiental; sobre todo, para mejorar las condiciones de vida de la población amazónica. Ese es el gran debate. La propuesta oficial está en los primeros puntos y va perdiendo prioridad hacia abajo. Nosotros queremos que estos recursos prioritariamente deban servir para la restauración de la naturaleza en la Amazonía, sin que eso implique que a la Chevron-Texaco se le exima de pagar lo que destruyó.

Una opción inteligente de inversión de los recursos obtenidos dentro de esta iniciativa podría ser la recuperación y desarrollo de tecnologías alternativas propias por ejemplo en temas agua, de agricultura, de energías limpias, descentralizadas y de bajo impacto. Sólo superando la dependencia del patrón tecnológico, podremos realmente construir un camino distinto, el camino del buen vivir o Sumak Kawsay. Recordemos 
que gran parte de esas tecnologías propias se encuentra en los saberes ancestrales o en el ejercicio de la creatividad de las comunidades marginalizadas; a las que, en lugar de rescatarlas, se los condena al poner en riesgo sus territorios con grandes proyectos extractivos.

M. L. Q. ¿Existe el riesgo de que Ecuador siga siendo dependiente de la comunidad internacional para financiar su modelo alternativo de desarrollo?

$A$. A. No veo ese riesgo si se parte del principio de co-responsabilidad, $y$ de justicia ambiental. Ya que no se trata de una tradicional ayuda al desarrollo. No es una inversión extranjera directa. Además, el manejo soberano de los recursos está en manos del Estado ecuatoriano. Lo que requerimos es un mecanismo para viabilizar el fideicomiso de los recursos que se obtengan y luego su manejo adecuado y eficiente, controlado por la sociedad civil ecuatoriana e internacional. Esta propuesta no es un proyecto de desarrollo aislado.

Esta iniciativa ITT conlleva un mensaje muy fuerte: hay que cambiar radicalmente las formas de relacionarnos con la naturaleza. Basta de los discursos sobre los impactos derivados del calentamiento global y el cambio climático, el mundo requiere acciones concretas. Esta es una oportunidad que tiene el Ecuador y el mundo entero para encontrar respuestas creativas, audaces, revolucionarias. Entonces, a mí no me preocupa la supuesta dependencia financiera, si estamos partiendo de una base de co-responsabilidad, todos somos co-responsables del planeta. Pero unos países lo son mucho más porque han provocado mayores distorsiones y destrozos; y esos son los países industrializados.

M. L. Q. Si se da un cambio de gobierno, y el nuevo quiere extraer el petróleo de la reserva, ¿cuáles son los mecanismos para impedir esto?

A. A. Hay para comenzar una base constitucional que impide actividades petroleras en áreas protegidas y en territorios de pueblos aislados. Para hacerlo se requeriría de una autorización de la Asamblea Nacional y, eventualmente, de una consulta popular. Si ese fuera el caso habría que trabajar desde la sociedad para garantizar que esto no suceda.

Una garantía radica en los Certificados de Garantía Yasuní. Se ha previsto que Ecuador venda dichos certificados, los que podrían generar entre 5.000 a 6.000 millones de dólares. Si Ecuador empieza con las acti- 
vidades para extraer el petróleo del ITT, automáticamente pierde este fondo y se queda sin los ingresos que generaría el mismo. Ese fondo revertiría a los países, a las personas que dieron el dinero. Eso es una garantía.

Además, el gobierno que tome esa desacertada decisión deberá considerar que desde que empiezan las actividades de exploración hasta que fluya el primer barril de petróleo pasan varios años. En concreto, en ese lapso Ecuador no tendría los ingresos de dicho fideicomiso, ni los que se generarían por la exportación de crudo.

Otra garantía: si se extrae el petróleo, ese petróleo extraído debería, a mi criterio, pasar a ser propiedad de las personas naturales y jurídicas que aportaron para financiar el fondo. Pero aquí hay dos problemas. El primero es que el gobierno ecuatoriano puede decir "yo no estoy exportando este petróleo. Este petróleo, lo estoy usando adentro, el que exporto es de otro campo, es de la empresa privada”. El segundo es que puede llegarse incluso a una situación en la que ya no exportamos petróleo, sino que somos importadores de petróleo. Entonces no habría el petróleo para embargar. Además, en Ecuador hay un problema constitucional. Nosotros no podemos disponer de las reservas porque el petróleo es un bien del Estado, un recurso inembargable, inejecutable, inalienable.

Entonces, hay que buscar un mecanismo para que ese crudo se quede en el subsuelo para siempre. Por eso es importante el compromiso de la comunidad internacional $y$, por cierto, de la sociedad ecuatoriana.

Debe haber una garantía adicional. Nosotros proponemos una veeduría internacional para que se use adecuadamente el dinero obtenido, de ninguna manera para la compra de armas o para actividades extractivas, minería por ejemplo, porque sería irracional.

\section{L. Q. ¿Cómo se podría extender este ejemplo a los demás países del Sur?}

A. A. En el Protocolo de Kioto, en el anexo 1: los países ricos tienen que bajar las emisiones -Europa, Estados Unidos (aunque no lo haya ratificado), Japón-. Luego, en el anexo 2 están los otros países que no tienen obligaciones. Un tema pendiente son las responsabilidades que las economías emergentes deberán asumir, como es el caso de China, Brasil, Rusia, Corea del Sur, que están contaminando mucho.

Nosotros estamos planteando la constitución del anexo 0. Es decir, países que llevan adelante enormes 
esfuerzos para proteger la atmósfera, como Ecuador al dejar el crudo en tierra en el ITT. Estos países deberían tener un tratamiento preferencial en términos de comercio internacional, de finanzas internacionales.

Habría que ver cómo se da paso a una multiplicación de este tipo de proyectos. Yo creo que es un ejercicio muy interesante.

La interrogante que surge es iA dónde vamos llegando con todo esto? Claro que puede generar algunos problemas. Por ejemplo, desde la lógica de una persona en la Unión Europea, ante la posibilidad de que se generalice este tipo de proyectos, se podría preguntar ¿con qué hago funcionar mi automóvil?, ¿qué quemó para hacer funcionar la calefacción de mi casa?, ¿quién sostiene mi bienestar? Aquí surge el meollo de la iniciativa ITT. No se trata sólo de dejar el crudo en el suelo y conseguir todos los objetivos planteados anteriormente, muy válidos por cierto. Lo que se busca es cambiar el estilo de vida de todos los habitantes en el planeta. No serán necesarios tantos automóviles, se requerirá más transporte público, menos contaminante. Se requerirán vehículos de transporte público que funcionen con electricidad, que no sea generada con petróleo, ni carbono o energía nuclear. ¿A dónde vamos llegando? A pensar en un mundo diferente, donde el respeto a la vida esté en el centro.

M. L. Q. En los países occidentales hablamos del bienestar. Aquí se habla de Sumak Kawsay o "Buen vivir", conceptos que vienen principalmente del movimiento indígena y que están reconocidos en la Constitución ecuatoriana. ¿Puedes explicar estos conceptos y definir la diferencia entre "bienestar" y "buen vivir"?

A. A. El bienestar y el buen vivir son conceptos diferentes. Son conceptos que merecen ser aclarados. Nosotros, en la Constituyente de Montecristi, hace ya más de un año, discutimos estos temas e impulsamos cambios abriendo la puerta al debate. El punto de partida fue reconocer los aportes culturales de los pueblos y nacionalidades indígenas. En Ecuador, los Kichwas hablan del Sumak Kawsay. En Bolivia, los Aymaras hablan de Suma Qamaña. Son visiones del mundo que buscan una mayor armonía del ser humano consigo mismo, con sus congéneres y con la naturaleza. Esa es una visión que surge de estas propuestas indígenas.

Dicho lo anterior, entendamos que en la comprensión del sentido 
que tiene y debe tener la vida de las personas, en las sociedades indígenas no existe el concepto de desarrollo. Es decir, no hay la concepción de un proceso lineal que establezca un estado anterior o posterior. No hay aquella visión de un estado de subdesarrollo a ser superado. Y tampoco un estado de desarrollo a ser alcanzado. No existe, como en la visión occidental, está dicotomía que explica y diferencia gran parte de los procesos en marcha. Para los pueblos indígenas tampoco hay la concepción tradicional de pobreza asociada a la carencia de bienes materiales o de riqueza vinculada a su abundancia.

Desde la cosmovisión indígena el mejoramiento social - ¿su desarrollo?- es una categoría en permanente construcción y reproducción. En ella está en juego la vida misma. Siguiendo con este planteamiento holístico, por la diversidad de elementos a los que están condicionadas las acciones humanas que propician el "Buen Vivir", los bienes materiales no son los únicos determinantes. Hay otros valores en juego: el conocimiento, el reconocimiento social y cultural, los comportamientos éticos e incluso espirituales en la relación con la sociedad y la naturaleza, los valores humanos, la visión de futuro, entre otros. "El Buen Vivir" aparece como una categoría en la filosofía de vida de las sociedades indígenas ancestrales, que va perdiendo terreno por efecto de las prácticas y mensajes de la modernidad occidental. Su aporte, sin embargo, sin llegar a una equivocada idealización, nos invita a asumir otros saberes y otras prácticas.

Pero la visión andina no es la única fuente de inspiración para impulsar el "Buen Vivir". Incluso desde círculos de la cultura occidental se levantan cada vez más voces que podrían estar de alguna manera en sintonía con esta visión indígena y viceversa. En el mundo se comprende, paulatinamente, la inviabilidad global del estilo de desarrollo dominante.

Frente a los devastadores efectos de los cambios climáticos, se plantean transformaciones profundas para que la humanidad pueda escapar con vida de los graves riesgos ecológicos y sociales en ciernes. El crecimiento material sin fin podría culminar en un suicidio colectivo, tal como parece augurar el mayor recalentamiento de la atmósfera o el deterioro de la capa de ozono, la pérdida de fuentes de agua dulce, la erosión de la biodiversidad agrícola y silvestre, la degradación de suelos o la propia desaparición de espacios de vida de las comunidades locales...

Para empezar, el concepto mismo de crecimiento económico debe ser 
reubicado en una dimensión adecuada. Crecimiento económico no es sinónimo de desarrollo.

Desde esas perspectivas múltiples planteamos la idea del "Buen Vivir" o Sumak Kawsay, como una oportunidad de ser construida. En definitiva no hay una definición rígida del buen vivir. La estamos construyendo en el mundo, no sólo en Ecuador.

Lo que si sabemos es que aquí no nos interesa el bienestar tradicional entendido como la acumulación de bienes materiales. Tampoco buscamos el bienestar dominando a la naturaleza, imponiéndonos a nosotros sobre la naturaleza. Esa lógica del bienestar, para nosotros, no existe. Entonces, aquí, incluso, tendremos que comenzar a elaborar nuevos indicadores para leer como avanzamos en esta idea del "Buen Vivir".

\section{L. Q. Este proyecto forma parte de} una multitud de medidas que adoptó el gobierno de Correa para luchar contra la pobreza, el cambio climático y para intentar cambiar el sistema de desarrollo. ¿Cuáles son estas otras medidas?

A. A. Ecuador ha asumido un liderazgo internacional en varios temas, muchos de ellos relacionados con la soberanía. Somos, por ejemplo, un territorio de paz, sin bases militares extranjeras. Hemos promovido una agenda de visibilización de las deudas ilegítimas. Desconocimos al CIADI, aquel sistema de arbitraje dependiente del Banco Mundial, como instancia de arbitraje internacional que protege a las inversiones de las transnacionales. Hemos sido importantes promotores de una nueva integración regional.

Somos pioneros en el reconocimiento de los derechos de la naturaleza.

Ha habido otras señales muy importantes. Por ejemplo, en la OPEP el presidente Correa planteó la necesidad de introducir un impuesto a cada barril de petróleo que salga del subsuelo. Eso ya lo había propuesto conceptualmente Herman Daly, un economista ecológico que trabajó muchos años en el Banco Mundial. Correa tuvo el mérito de plantearlo políticamente. Lamentablemente, hasta ahora ha quedado como una propuesta interesante y no ha prosperado. Este impuesto permitiría tener un fondo en el mundo entero para financiar el uso de energías alternativas, renovables, para transitar hacia una economía pospetrolera, indispensable en el planeta. Recordemos que ese es uno de los objetivos del proyecto ITT: caminar hacia una economía pospetrolera, una economía que no dependa tanto del petróleo. 
Y luego también, el gobierno del presidente Correa está apoyando la iniciativa del Banco del Sur, del fondo de reservas latinoamericano relacionado con el SUCRE (Sistema Único de Compensación Regional). Apoyó también la iniciativa del Tribunal Internacional de Arbitraje de Deuda Soberana. Sintetizando, estos esfuerzos son el resultado de las luchas de resistencia y de construcción colectiva desde la sociedad ecuatoriana. Se avanza en algunos aspectos. El gobierno, recogiendo esas iniciativas colectivas, abre puertas, pero por igual hay que lamentar que este mismo gobierno es incapaz de abrir el debate sobre el futuro de la economía extractivista, que al parecer se reproduciría si se sostiene la posibilidad para inversiones en el ámbito de la minería metálica a gran escala y a cielo abierto, tal como plantea la Ley de Minería aprobada a inicios de este año [2009] por parte del mismo gobierno que alienta la iniciativa ITT. 